\title{
Detecting Novel Features of an Environment Using Habituation *
}

\author{
Stephen Marsland Ulrich Nehmzow Jonathan Shapiro \\ Department of Computer Science \\ University of Manchester \\ Oxford Road \\ Manchester M13 9PL \\ \{smarsland, ulrich, jls\}@cs.man.ac.uk
}

\begin{abstract}
In this paper a novelty filter is introduced which allows a robot operating in an unstructured environment to produce a self-organised model of its surroundings and to detect deviations from the learned model. The environment is perceived using the robot's 16 sonar sensors. The algorithm produces a novelty measure for each sensor scan relative to the model it has learned. This means that it highlights stimuli which have not been previously experienced. The novelty filter proposed uses a model of habituation. Habituation is a decrement in behavioural response when a stimulus is presented repeatedly. Robot experiments are presented which demonstrate the reliable operation of the filter in a number of environments.
\end{abstract}

\section{Introduction}

The ability to detect and respond suitably to novel stimuli, that is new or changed features within an environment, is very useful to animals and robots. Studies have shown that animals can rapidly recognise changes in their environment (O'Keefe and Nadel, 1977, Knight, 1996). This ability has two main purposes - to avoid predators and to find potential food (Pribram, 1992). This paper describes an algorithm suitable for detecting novel stimuli and applies it to an autonomous agent. The filter learns to recognise features which it has seen before and evaluates the novelty of sonar scans taken as a robot explores an environment.

Experiments are presented which demonstrate that the algorithm can learn an internal representation of one environment and then use this model in a second, similar, environment to detect new features which were not present in the first environment. The system could therefore be used in inspection tasks, being trained in a section of the environment that is known to be 'clean', i.e., containing no undesired features. Having learned in the 'clean' environment, the robot could move into the

*In From Animals to Animats, Proceedings of the Sixth International Conference on Simulation of Adaptive Behaviour, 2000 wider area, highlighting those features which were novel. These would be features which were not present in the original environment. A novelty filter can also be used to direct the attention of the robot to new stimuli, so that the amount of processing needed to deal with its sensory perceptions can be reduced. It can also be used to decide when a feature should be learned by recognising that it is new, that is, different to previous perceptions.

\subsection{Habituation}

Habituation - a reduction in behavioural response when a stimulus is perceived repeatedly - is thought to be a fundamental mechanism for adaptive behaviour. It is present in animals from the sea slug Aplysia (Bailey and Chen, 1983, Greenberg et al., 1987) to humans (O'Keefe and Nadel, 1977) through toads (Ewert and Kehl, 1978, Wang and Arbib, 1992) and cats (Thompson, 1986). In contrast to other forms of behavioural decrement, such as fatigue, a change in the nature of the stimulus restores the response to its original level - a process known as dishabituation. In addition, if a particular stimulus is not presented for a period of time, the response is recovered, a form of 'forgetting'. An overview of the effects and causes of habituation can be found in Thompson and Spencer, 1966, Peeke and Herz, 1973).

A number of researchers have produced mathematical models of the effects of habituation on the efficacy of a synapse. They include Groves and Thompson, Stanley, and Wang and Hsu (Groves and Thompson, 1970, Stanley, 1976, Wang and Hsu, 1990). The models are similar, except that Wang and Hsu's allows for long term memory, while the others do not. Long term memory means that an animal habituates more quickly to a stimulus to which it has habituated previously. For simplicity, the model which is used in the work presented here is that of Stanley. In his model the synaptic efficacy, $y(t)$, decreases according to the following equation:

$$
\tau \frac{d y(t)}{d t}=\alpha\left[y_{0}-y(t)\right]-S(t)
$$

where $y_{0}$ is the original value of $y, \tau$ and $\alpha$ are time 


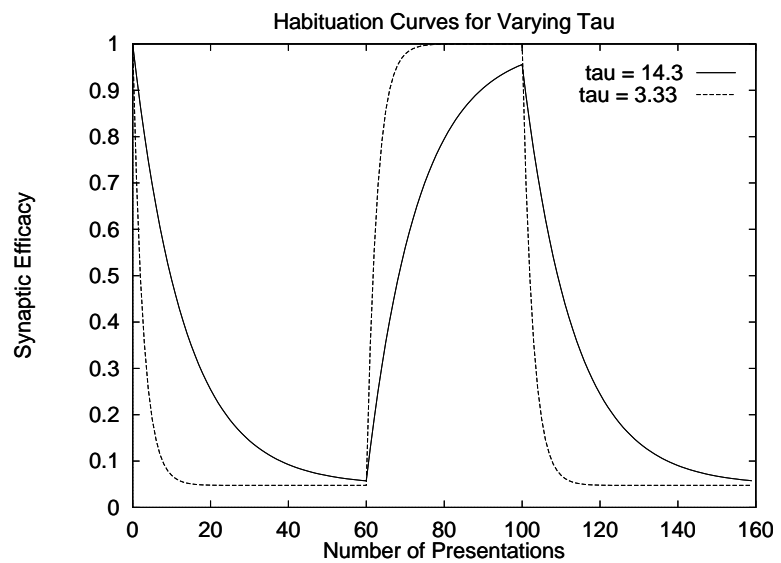

Figure 1: An example of how the synaptic efficacy drops when habituation occurs. In both curves, a constant stimulus $S(t)=1$ is presented, causing the efficacy to fall. The stimulus is reduced to $S(t)=0$ at time $t=60$ where the graphs rise again, and becomes $S(t)=1$ again at $t=100$, causing another drop. The two curves show the effects of varying $\tau$ in equation 1 . It can be seen that a larger value of $\tau$ causes both the learning and forgetting to occur faster. The other variables were the same for both curves, $\alpha=1.05$ and $y_{0}=1.0$.

constants governing the rate of habituation and recovery respectively, and $S$ is the stimulus presented. A graph of the effects of the equation can be seen in figure 1 .

\subsection{Novelty Detection}

Habituation can be used to detect novel stimuli in a very simple way. A series of habituating neurons can be used to build an internal representation of perceived stimuli, and then stimuli not included in this model will be the only ones which reach the attention of the animal. It has been suggested Heiligenberg, 1980, Grau and Bastian, 1986) that pulse-type weakly electric fish use a mechanism similar to this when they use the returns from weak electric pulses to sample their environment. Heiligenberg (Heiligenberg, 1980) suggests that the fish store a 'template' of responses received from their environment, and detect novelty with respect to this memory, changing the template if novel stimuli remain for several samples.

\subsection{Related Work}

A number of novelty detection techniques have been proposed in the neural network literature. Most of them are trained off-line. For example, the Kohonen Novelty Filter (Kohonen and Oja, 1976, Kohonen, 1993), is an autoencoder neural network trained using backpropagation of error, so that the network extracts the principal components of the input. After training, any input presented to the network produces one of the learned outputs, and the bitwise difference between input and output highlights novel components of the input. Several authors have investigated the effects of this filter, notably Aeyels (Aeyels, 1990) who added a 'forgetting' term into the equations.

Other approaches include that of Ho and Rouat (Ho and Rouat, 1998), who used an integrate-and-fire model inspired by layer IV of the cortex. Their model measures how long an oscillatory network takes to converge to a stable solution, reasoning that previously seen inputs should converge faster than novel ones.

Several researchers have used the gated dipole (Grossberg, 1972), a construct which compares current and recent values of a stimulus. Levine and Prueitt (Levine and Prueitt, 1992 used it to compare inputs with pre-defined stimuli, novel inputs causing greater output values. They proposed a model where a number of gated dipoles were linked into a 'dipole field' and used it to model novelty preference in frontally lesioned rhesus monkeys. The gated dipole has also been used by Öğmen and Prakash (Oğmen and Prakash, 1997) who used it as part of a system to control a robot arm, which moved its end effector to places which were novel. This was done by quantising the workspace and associating a gated dipole with each separate area. Their work also considered the question of detecting novel objects. They did this by taking the output of an ART network (Carpenter and Grossberg, 1988), which attempts to classify the objects into a number of categories, and feeding it into a network of gated dipoles.

Ypma and Duin (Ypma and Duin, 1997) proposed a novelty detection mechanism based on the self-organising map. The distance of the winning neuron from neighbourhoods which have fired recently was calculated, and those beyond a certain threshold were counted as novel. This method was used by Taylor and MacIntyre (Taylor and MacIntyre, 1998) to detect faults when monitoring machines. The network was trained on data taken from machines operating normally, and data deviating from this pattern was taken as novel. This is a common technique when faced with a problem for which there is very little data in one class, relative to others. Examples include machine breakdowns Nairac et al., 1999, Worden et al., 1999) and mammogram scans (Tarassenko et al., 1995). Often supervised techniques such as Gaussian Mixture Models or Parzen Windows are used, and the problem reduces to attempting to recognise when inputs do not belong to the distribution which generates the normal data (Bishop, 1994). The method proposed by Ypma and Duin relies very strongly on the choice of threshold and on the properties of the data presented to the network, which must form strictly segmented neighbourhood clusters without much spread. 


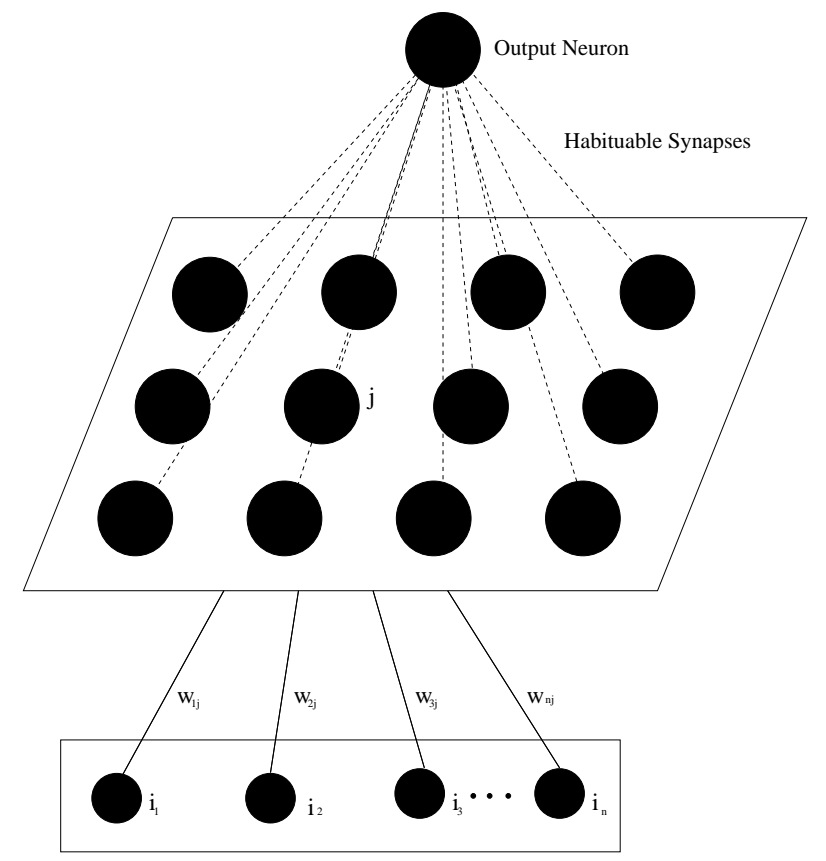

Figure 2: The novelty filter. The input layer connects to a clustering layer which represents the feature space, the winning neuron (i.e., the one 'closest' to the input) passing its output along a habituable synapse to the output neuron so that the output received from a neuron reduces with the number of times it fires.

Growing networks such as Adaptive Resonance Theory (ART) Carpenter and Grossberg, 1988 can be used to define as novel those things which have never been seen before, by using a new, uncommitted node to represent them. The use of habituation allows novelty to be defined more specifically as those things which have not been seen in the current context. The filter does this by learning an online, adaptive representation of the current environment. Habituation also allows the novelty of a stimulus to be evaluated, so that the novelty reduces with perception over time. The combination of the two ideas, amalgamating growing networks with habituation has been considered in (Marsland et al., 2000).

\section{The Habituating Self-Organising Map (HSOM)}

This section introduces the novelty filter on which this work is based. An input vector is presented to a clustering network, which finds a winning neuron using a winner-takes-all strategy. Each neuron in the map field is connected to the output neuron (see figure 2) via a habituable synapse, so that the more frequently a neuron fires, the lower the efficacy of the synapse and hence the lower the strength of the output. The behaviour of the habituable synapses is controlled by equation 1 . The strength of the winning synapse is taken as a nov- elty value for the particular winning neuron, and hence the perception presented, with more novel stimuli having values closer to 1 , and more common stimuli values closer to 0 .

There are two choices of how to deal with the output synapses of neurons which do not belong to the winning neighbourhood. They could be left without any input, so that they do not habituate and their value remains unchanged, or, instead, a zero input $(S(t)=0)$ could be given. In this case the synapses will 'forget' previous inhibition over time, with the time controlled by the constant $\tau$. This can be seen in the second, ascending part of figure 1. In the results presented here the network remembers all perceptions, forgetting is not used and the synapses of neurons which are not in the winning neighbourhood do not receive any input.

In the implementation described below, a Kohonen Self-Organising Map (SOM) implementing Learning Vector Quantisation Kohonen, 1993 is used as the clustering mechanism. Kohonen networks are often used for problems dealing with robot sensory inputs (Kurz, 1996). Although guaranteeing convergence of a two dimensional network is still an unsolved problem (Erwin et al., 1992), the network performs a useful dimensionality reduction and clusters the perceptions in this lower dimensional space. The network calculates the distance between the input and each of the neurons in the map field where the distance is defined by:

$$
d=\sum_{i=0}^{N-1}\left(\mathbf{w}_{i}(t)-\mathbf{v}(t)\right)^{2},
$$

where $\mathbf{v}(t)$ is the input vector at time $t, \mathbf{w}_{i}$ the weight between input $i$ and the neuron and the sum is over the $N$ components of the input vector. The neuron with the minimum $d$ is selected and the weights for that neuron and its eight topological neighbours are updated by:

$$
\mathbf{w}_{i}(t+1)=\mathbf{w}_{i}(t)+\eta(t)\left(\mathbf{v}(t)-\mathbf{w}_{i}(t)\right)
$$

where $\eta$ is the learning rate, $0 \leq \eta(t) \leq 1$. The habituable synapses for both the winning neuron and its topological neighbours were updated, using equation 1 with $S(t)=1$. The value of $\tau$ used was different for the winner and its neighbours, being $\tau=3.33$ for the winner and $\tau=14.3$ for the neighbourhood synapses. This meant that winning neurons habituated quickly, while neighbourhood neurons, which recognise similar perceptions, have a smaller amount of habituation. The effects of these values can be seen in figure it. The synapses of other neurons were not affected. Other variables kept their values regardless of which neuron is firing, being $\alpha=1.05$ and $y_{0}=1.0$. With these values the synaptic efficacy never falls below 0.0476 (see figure 1), a function of the values used for the constants in equation 1 . 


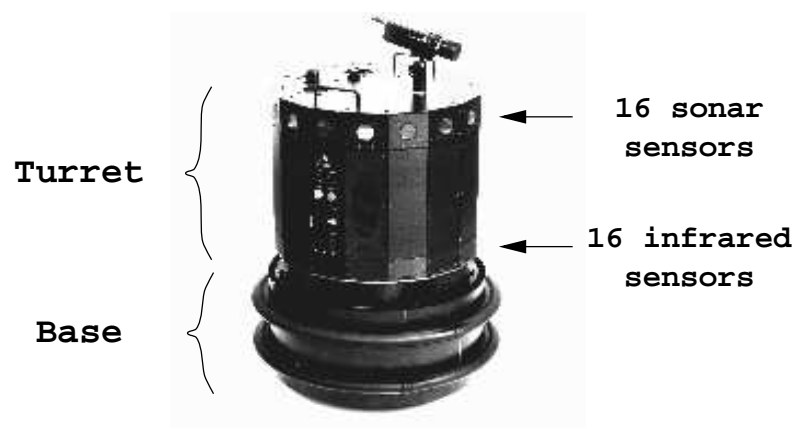

Figure 3: The Nomad 200 mobile robot.

A square map field, comprising 100 neurons arranged in a 10 by 10 grid, was used. The neighbourhood size was kept constant at \pm 1 unit in all directions and the learning rate $\eta$ was 0.25 , so that the network was always learning.

\section{Experiments}

The experiments presented investigate the ability of the novelty filter to learn a model of an external environment through periodic sonar scans taken whilst exploring, and to detect deviations from that model.

\subsection{The Robot}

A Nomad 200 mobile robot (shown in figure 3) was used to perform the experiments. The band of infra-red sensors mounted at the bottom of the turret of the robot were used to perform a pre-trained wall-following routine (Nehmzow, 1994), and the 16 sonar sensors at the top of the turret were used to provide perceptions of the robot's environment. The angle between the turret and base of the robot was kept fixed. The input vector to the novelty filter consisted of the 16 sonar sensors, each normalised to be between 0 and 1 and thresholded at about 4 metres. The readings were inverted so that inputs from sonar responses received from closer objects were greater.

\subsection{Description of the Experiments}

Each experiment consisted of a number of trials, with each trial taking the same form. The robot was positioned at a starting point chosen arbitrarily within the environment. From this starting point the robot travelled for 10 metres using a wall-following behaviour, training the HSOM as sensory stimuli were perceived, and then stopped and saved the weights of the HSOM. Approximately every $10 \mathrm{~cm}$ along the route the smoothed values of the sonar perceptions over that $10 \mathrm{~cm}$ of travel were presented to the novelty filter, which produced a novelty value for that perception. At the end of the run a manual control was used to return the robot to the beginning, and the same procedure repeated, starting with the weights learned during the previous run.

The trials were performed in pairs. After every training run, where the sonar readings were used to train the weights of the HSOM, a second, non-learning, trial was performed. In this run the sonar inputs generated outputs from the novelty filter, which records how novel the perceptions were. At the beginning of the first trial every perception was novel, as they had not been perceived before, but after a short distance the filter began to recognise some similar perceptions, and after a number of runs in an environment an accurate representation had been reached so that no perceptions were seen as novel, i.e., the output of the novelty filter was only the resting activity of the output neuron throughout the run. Once this occurred the environment was changed. This could either be a modification of the current environment (such as the opening of a door), or the robot could be moved to a new environment. The changes are described in section 1 .

\subsection{Environments}

Three different environments were used, together with a control environment for training, as shown in figure 4 . Environments A and B are two sections of corridor on the second floor of the Computer Science building at the University of Manchester. The corridors are $1.7 \mathrm{~m}$ wide and have walls made from painted breezeblock. Doors made of varnished wood lead from the corridors into offices. Environment $\mathrm{C}$ is part of the first floor of the building. It is a section of corridor $2.1 \mathrm{~m}$ wide, with smooth brick on one side and a wall of glass on the other. This environment has display boards mounted on the wall, as can be seen in figure 4 . These boards are sufficiently low to be visible to the sonar sensors. They are made from a laminated, shiny plastic. In all the experiments the environments were kept static.

\section{Results}

\subsection{Environment $A$}

In the first experiment, shown in figure 5, the robot learned a representation of environment A (see figure 4), using the procedure described in section 3.2, so that it no longer detected any novelty when traversing it by following the wall. The left side of figure 5 shows the sequence of trials in which the robot learned an internal representation of environment A. In the first trial the novelty filter is learning, as can be seen from the characteristic habituation shape of successive readings near the beginning of the run and where the door on the right of the robot is perceived (at about 3 metres). It can be seen that everything is initially novel, but the filter rapidly habituates to the brick wall. In all the experiments the 

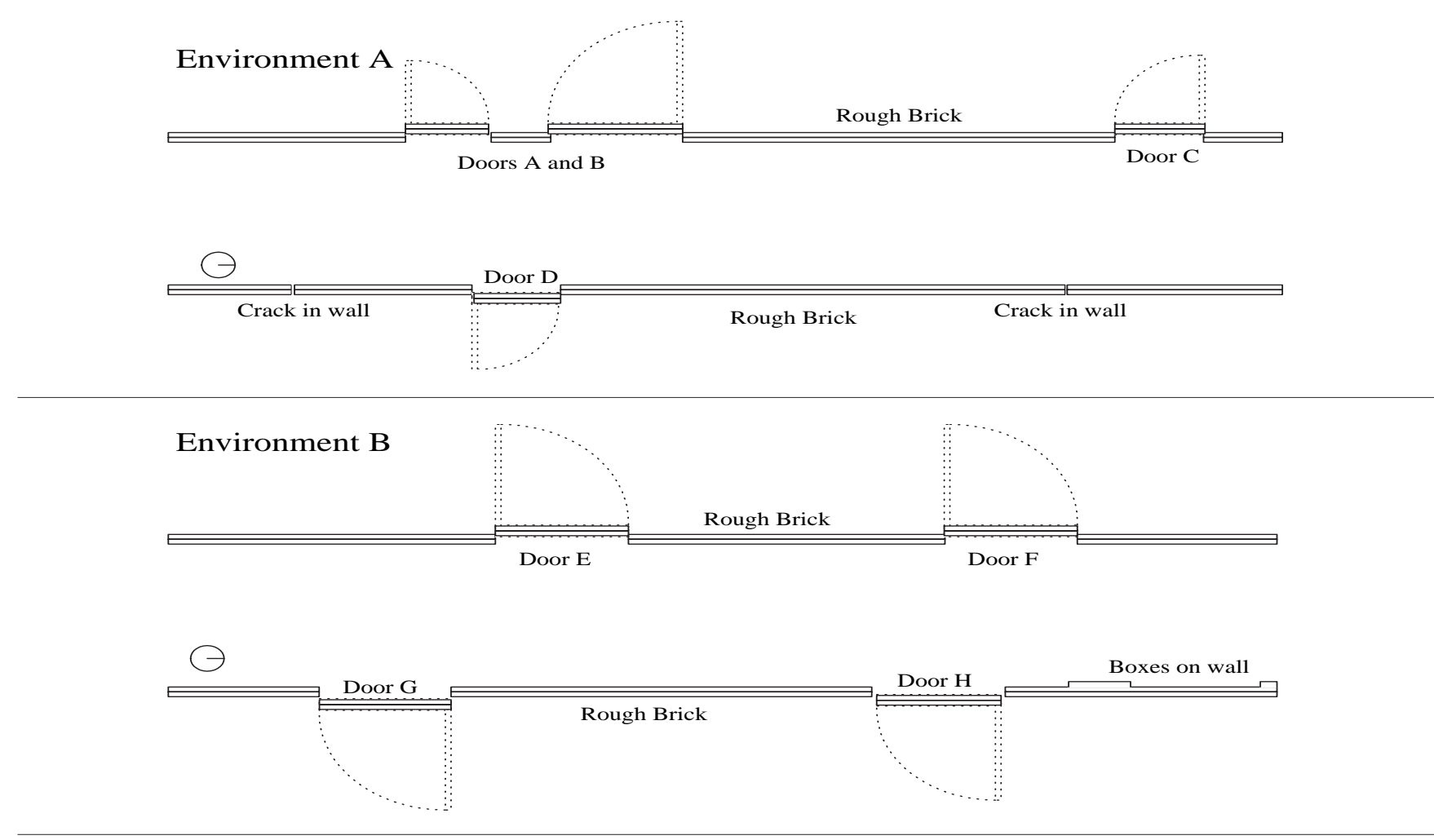

\section{Environment $\mathrm{C}$}

Windows

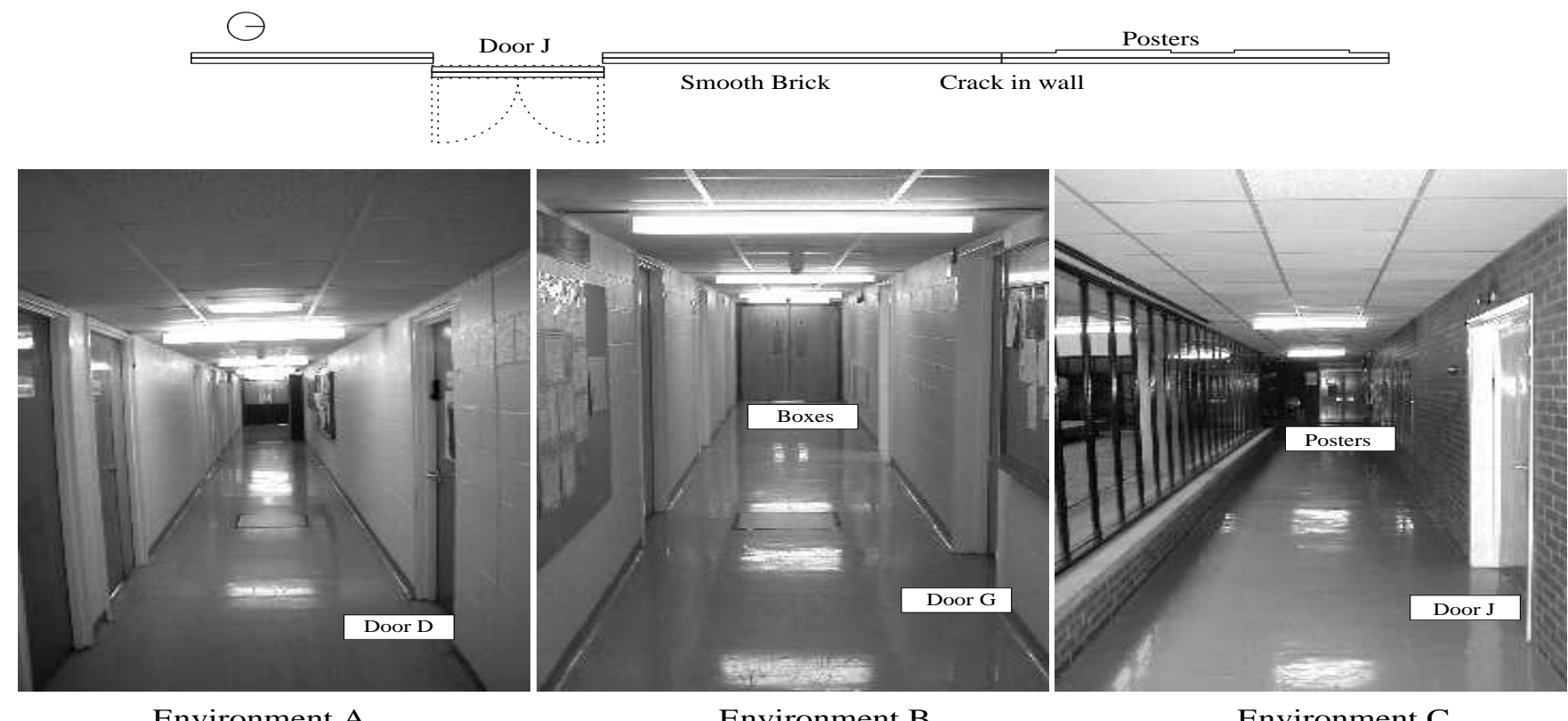

Environment A

Environment B

Environment C

Figure 4: Diagrams of the three environments used. The robot is shown facing in the direction of travel adjacent to the wall that it followed. Environments $A$ and $B$ are two similar sections of corridor, while environment $C$ is wider and has walls made of different materials, glass and brick instead of breezeblock. The photographs show the environments as they appear from the starting position of the robot. The notice boards which are visible in environment B are above the height of the robot's sonar sensors, and are therefore not detected. 


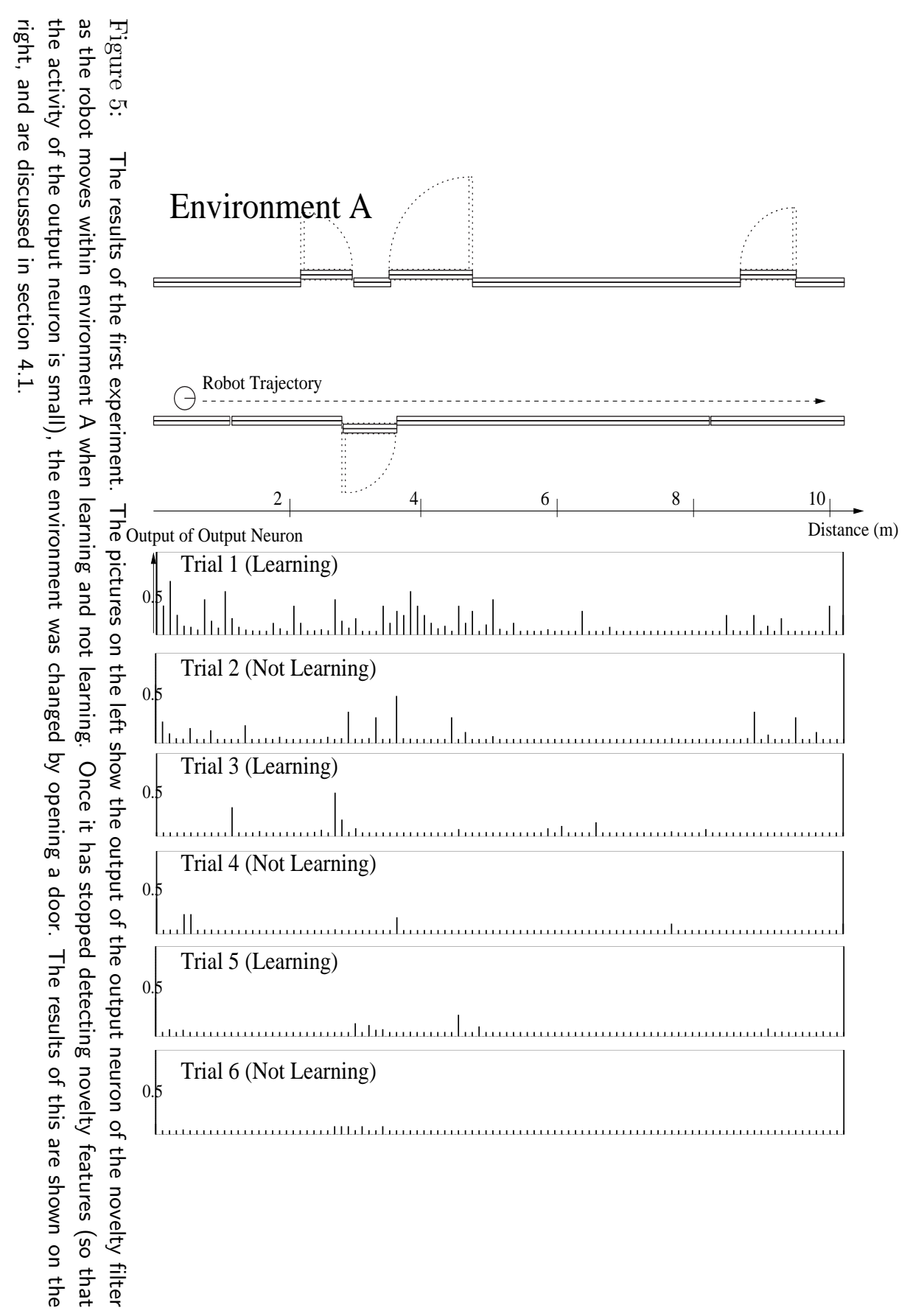

\section{Environment A* (identical to A apart from open door)}


Trial 4 (Learning)

0.5

0.5 


\begin{tabular}{|c|c|c|c|}
\hline \multirow{2}{*}{$\begin{array}{c}\text { Exp } \\
\text { (Env) }\end{array}$} & \multirow[t]{2}{*}{ Trial } & \multicolumn{2}{|c|}{ Output of the Output Neuron } \\
\hline & & Integrated & Maximum \\
\hline \multirow[t]{7}{*}{$1(\mathrm{~A})$} & & & \\
\hline & $1(\mathrm{~L})$ & 9.17 & 1.00 \\
\hline & $2(\mathrm{NL})$ & 2.64 & 0.53 \\
\hline & $3(\mathrm{~L})$ & 1.34 & 0.43 \\
\hline & $4(\mathrm{NL})$ & 1.07 & 0.42 \\
\hline & $5(\mathrm{~L})$ & 0.52 & 0.42 \\
\hline & $6(\mathrm{NL})$ & 0.30 & 0.12 \\
\hline \multirow[t]{6}{*}{$1\left(A^{*}\right)$} & & & \\
\hline & $1(\mathrm{NL})$ & 1.44 & 0.65 \\
\hline & $2(\mathrm{~L})$ & 1.45 & 0.65 \\
\hline & $3(\mathrm{NL})$ & 0.37 & 0.17 \\
\hline & $4(\mathrm{~L})$ & 0.14 & 0.08 \\
\hline & $5(\mathrm{NL})$ & 0.01 & 0.05 \\
\hline \multirow[t]{7}{*}{$2(\mathrm{~B})$} & & & \\
\hline & $1(\mathrm{NL})$ & 3.38 & 0.65 \\
\hline & $2(\mathrm{~L})$ & 1.18 & 0.42 \\
\hline & $3(\mathrm{NL})$ & 1.21 & 0.42 \\
\hline & $4(\mathrm{~L})$ & 0.87 & 0.35 \\
\hline & $5(\mathrm{NL})$ & 0.03 & 0.08 \\
\hline & Control & 27.09 & 1.00 \\
\hline \multirow[t]{6}{*}{$3(\mathrm{C})$} & $1(\mathrm{NL})$ & 9.68 & 0.65 \\
\hline & $2(\mathrm{~L})$ & 2.56 & 0.65 \\
\hline & $3(\mathrm{NL})$ & 3.06 & 0.53 \\
\hline & $4(\mathrm{~L})$ & 0.98 & 0.42 \\
\hline & $5(\mathrm{NL})$ & 0.29 & 0.15 \\
\hline & Control & 27.75 & 1.00 \\
\hline
\end{tabular}

Table 1: The table shows the integrated output of the output neuron over each trial and the maximum output of the neuron. The integrated output has had the resting activity of the neuron ( 0.0476 for each time step) subtracted. It can be seen that both the integrated output and maximum output drop after each learning trial (labelled L).

robot gets a much clearer picture of the right hand side of the environment because that is the wall that it was following. The figures show the strength of output from the output neuron of the HSOM at each step along the 10 metre section of corridor which make up each environment.

In the second trial, where the filter is not learning, simply using the weights learned during the first, there is much less novelty. It is interesting to note that the crack in the wall near the beginning of the environment is perceived during the first and third trial, but not the second. This is because the crack is very thin and the perceptions of the robot depend on the speed at which it moves and its precise position. The features which take longer to learn are those which are perceived less frequently. For instance, the robot rapidly learns to recognise the perception of walls, but doorways, which are seen only occasionally, and from many different angles, take longer. This demonstrates that novel features are those which have been seen only infrequently, if at all.

In trial 6 it can be seen that the filter has learned a representation of the environment, because the output of the filter does not rise above the baseline resting activity of the output neuron. At this point the environment was altered by opening a door so that the sonar perception changed (shown as Environment $\mathrm{A}^{*}$ on the right hand side of figure 5). In order to prevent the robot from going through the door a cardboard box was placed in the doorway. This was of sufficient height to appear on the infra-red sensors, which were used for wall-following, but did not appear on the sonar scans. The novelty filter detects the open door, as can be seen from the burst of novelty in the figure, but successive trials show that the filter quickly learns to recognise this new feature. No other novel features were detected. It can be seen that the doorways are detected slightly before the robot reaches them and still detected for a small distance afterwards This is because the sonar scans project in front and behind of the robot.

\subsection{Environment $B$}

For the second experiment, shown on the left hand side of figure 6. the robot was put into environment B (shown in figure 4 ) and the novelty filter was initialised with the weights gained after the representation of environment A had been learned. Environment B was a very similar corridor environment to environment $\mathrm{A}$. The procedure of the first experiment was repeated, with the robot exploring a 10 metre section of the environment and then stopping. By comparing the integrated outputs of the output neuron in table 1 in environments $\mathrm{A}$ and $\mathrm{B}$ and the left hand side of figures and 6 , it can be seen that much less novelty was detected in the second environment than in the first. It can be seen that during the first trial in environment $\mathrm{B}$, shown on the left of figure 6 , the only place where the output of the neuron is especially high (signifying novelty) is at the first of the two doors on the right hand side of the robot (door G in figure (1). On inspection of the environment, it was discovered that this doorway was inset deeper than those in the first corridor.

The bottom graph on the left hand side of figure 6 shows the novelty values when the robot is put into environment $\mathrm{B}$ after training in a different environment. The environment used for the training was a large, open environment. The robot was steered about a course so that it moved along close to a wall, turned and moved into the middle of the environment and then returned to the wall. Both the graph and table 1 show that much more novelty was detected when the network trained in this control environment was moved into environment B. 




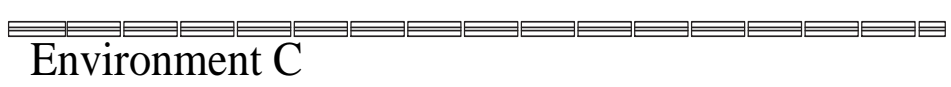

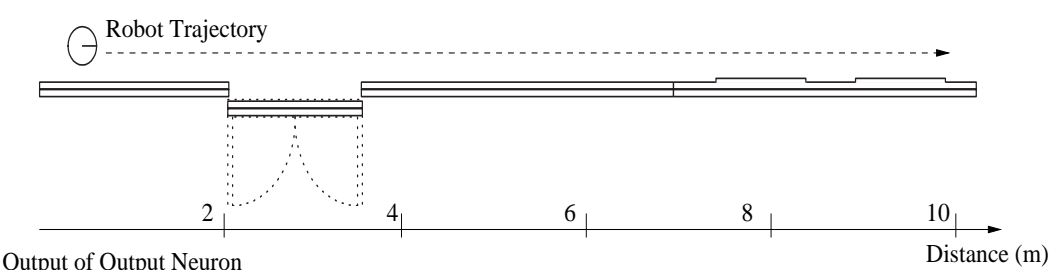

Output of Output Neuron Distance (m)


0.5 Trial 5 (Not Learning)

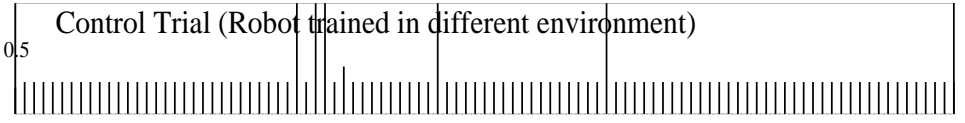




\subsection{Environment $C$}

The third experiment took the same form as the second, again starting from the weights gained during training in environment A. Using these weights the robot explored the environment labelled $\mathrm{C}$ in figure 1 , the results of which are shown on the right of figure 6 . Table 1 shows that much more novelty is detected in environment $\mathrm{C}$ than in environment $\mathrm{B}$ when the robot first explores it. This is to be expected because the environment is significantly different, being wider and having walls made of different materials (see the pictures at the bottom of figure (4). In particular, the doorway, which is particularly deeply inset, causes a high output from the HSOM, as do the posters at the end of the environment. Again, the control trial shows that the novelty filter detects some similarity between environments $\mathrm{C}$ and $\mathrm{A}$, which are both corridor environments but not between $\mathrm{C}$ and the open area in which the control filter was trained. The crack in the wall (at about the $7 \mathrm{~m}$ mark), which is a similar perception to that seen in environment A, was not detected.

\section{Summary and Conclusions}

A novelty filter has been proposed that uses habituation to assign a novelty value to perceptions. The filter learns on-line. The novelty filter has been demonstrated to be capable of learning a representation of features of an environment whilst running on-line on a mobile robot. Experimental results show that the filter can accurately detect novel features of the environment as deviations from the learned model. The effects of training the filter in one environment and then testing it in another show that if the two environments are similar, the filter accurately recognises the familiar features, and highlights new features. This is an adaptive behaviour, as both the network and habituation weights change so that the results of training the filter in one environment and then using it in another are different to simply using it in the second environment.

Future work will focus on two areas. The output of additional sensory systems will be added so that the filter gains more information about its environment, and alternative clustering mechanisms will be considered.

The current work showed that the filter works using sonar scans as inputs. Sonar readings are inherently noisy, but the novelty filter deals with this problem through the use of the self-organising map. In order to perceive more information about the environment, it will be necessary to integrate further sensor modes into the model. In particular, a monochrome CCD camera will be used. The images will have to be extensively preprocessed in order for the robot with its limited processing power to be able to deal with them sufficiently quickly. How the filter will deal with images, and how the sonar and image novelty filters will be integrated is currently under investigation. The vision system will be useful to give more information about the environment, and to make possible an analysis of the scene in order to clarify precisely which stimuli in a perception are novel.

Other work will investigate alternatives to the SelfOrganising Map as a suitable clustering mechanism. A number of well-documented problems with the SOM exist for problems like this, in particular the fact that the size of the network has to be decided before it is used and remains fixed. This is a problem because the network can become saturated so that previously learned perceptions are lost, or novel stimuli are misclassified as normal. These are aspects of the plasticity-stability dilemma Carpenter and Grossberg, 1988. Possible solutions include using a growing network such as the Growing Neural Gas of Fritzke (Fritzke, 1995). An alternative is to consider a Mixture of Experts (Jordan and Jacobs, 1994), with each expert being responsible for a particular type of perception and voting on its novelty. A committee of networks (Perrone and Cooper, 1993) could be used in a similar way.

Another interesting problem is how to deal with features which are spread out over time in the robot's perception. For example, for the robot travelling down a corridor, a door frame is always followed by a door. If the robot learns this, then if only one of this pair of perceptions is detected it will be considered novel. This is a question of temporal learning Chappell and Taylor, 1993, Euliano and Principe, 1998.

\section{Acknowledgements}

This research is supported by a UK EPSRC Studentship.

\section{References}

Aeyels, D. (1990). On the dynamic behaviour of the novelty detector and the novelty filter. In Bonnard, B., Bride, B., Gauthier, J., and Kupka, I., (Eds.), Analysis of Controlled Dynamical Systems, pages $1-10$.

Bailey, C. and Chen, M. (1983). Morphological basis of longterm habituation and sensitization in aplysia. Science, 220:91-93.

Bishop, C. M. (1994). Novelty detection and neural network validation. IEEE Proc Vision, Image and Signal Processing, 141(4):217-222.

Carpenter, G. A. and Grossberg, S. (1988). The ART of adaptive pattern recognition by a self-organising neural network. IEEE Computer, 21:77 - 88.

Chappell, G. and Taylor, J. (1993). The temporal Kohonen map. Neural Networks, 6:441-445.

Erwin, E., Obermayer, K., and Schulten, K. (1992). Selforganising maps: Ordering, convergence properties and energy functions. Biological Cybernetics, 67(1):47 - 55 . 
Euliano, N. and Principe, J. (1998). Temporal plasticity in self-organising networks. In Proceedings of the IEEE World Congress on Computational Intelligence, 1998.

Ewert, J.-P. and Kehl, W. (1978). Configurational preyselection by individual experience in the toad bufo bufo. Journal of Comparative Physiology A, 126:105-114.

Fritzke, B. (1995). A growing neural gas network learns topologies. In Tesauro, G., Touretzky, D. S., and Leen, T. K., (Eds.), Advances in Neural Information Processing Systems 7, pages 625-632, Cambridge, MA. MIT Press.

Grau, H. J. and Bastian, J. (1986). Neural correlates of novelty detection in pulse-type weakly electric fish. Journal of Comparative Physiology A, 159:191 - 200.

Greenberg, S., Castellucci, V., Bayley, H., and Schwartz, J. (1987). A molecular mechanism for long-term sensitisation in aplysia. Nature, 329:62-65.

Grossberg, S. (1972). A neural theory of punishment and avoidance. II. Quantitative theory. Mathematical Biosciences, 15:253-285.

Groves, P. and Thompson, R. (1970). Habituation: A dualprocess theory. Psychological Review, 77(5):419-450.

Heiligenberg, W. (1980). The evaluation of electroreceptive feedback in a gymnotoid fish with a pulse-type electric organ discharge. Journal of Comparative Physiology, 138:173-185.

Ho, T. V. and Rouat, J. (1998). Novelty detection based on relaxation time of a network of integrate-and-fire neurons. In Proceedings of the 2nd IEEE World Congress on Computational Intelligence, WCCI'98, pages 1524-1529.

Jordan, M. I. and Jacobs, R. A. (1994). Hierarchical mixtures of experts and the EM algorithm. Neural Computation, 6:181 - 214 .

Knight, R. T. (1996). Contribution of human hippocampal region to novelty detection. Nature, 383:256 - 259 .

Kohonen, T. (1993). Self-Organization and Associative Memory, 3rd ed. Springer, Berlin.

Kohonen, T. and Oja, E. (1976). Fast adaptive formation of orthogonalizing filters and associative memory in recurrent networks of neuron-like elements. Biological $\mathrm{Cy}$ bernetics, 25:85-95.

Kurz, A. (1996). Constructing maps for mobile robot navigation based on ultrasonic range data. IEEE Transactions on Systems, Man and Cybernetics - Part B: Cybernetics, 26(2):233-242.

Levine, D. S. and Prueitt, P. S. (1992). Simulations of conditioned perseveration and novelty preference from frontal lobe damage. In Commons, M. L., Grossberg, S., and Staddon, J. E., (Eds.), Neural Network Models of Conditioning and Action, chapter 5, pages 123 - 147. Lawrence Erlbaum Associates.

Marsland, S., Nehmzow, U., and Shapiro, J. (2000). Novelty detection for robot neotaxis. In Proceedings of the 2nd International Symposium on Neural Compuatation (NC'2000), pages $554-559$.
Nairac, A., Townsend, N., Carr, R., King, S., Cowley, P., and Tarassenko, L. (1999). A system for the analysis of jet system vibration data. Integrated Computer-Aided Engineering, 6(1).

Nehmzow, U. (1994). Autonomous acquisition of sensormotor couplings in robots. Technical Report UMCS94-11-1, Department of Computer Science, University of Manchester.

Öğmen, H. and Prakash, R. (1997). A developmental perspective to neural models of intelligence and learning. In Levine, D. S. and Elsberry, W. R., (Eds.), Optimality in Biological and Artificial Networks?, chapter 18, pages 363 - 395. Lawrence Erlbaum Associates, NJ.

O'Keefe, J. and Nadel, L. (1977). The Hippocampus as a Cognitive Map. Oxford University Press, Oxford, England.

Peeke, H. V. and Herz, M. J., (Eds.) (1973). Habituation, volume 1: Behavioural Studies. Academic Press.

Perrone, M. P. and Cooper, L. N. (1993). When networks disagree: Ensemble methods for hybrid neural networks. In Mammone, R., (Ed.), Neural Networks for Speech and Image Processing. Chapman-Hall.

Pribram, K. H. (1992). Familiarity and novelty: The contributions of the limbric forebrain to valuation and the processing of relevance. In Levine, D. S. and Leven, S. J., (Eds.), Motivation, Emotion and Goal Direction in Neural Networks, chapter 10, pages 337 - 365. Lawrence Erlbaum Associates.

Stanley, J. C. (1976). Computer simulation of a model of habituation. Nature, 261:146-148.

Tarassenko, L., Hayton, P., Cerneaz, N., and Brady, M. (1995). Novelty detection for the identification of masses in mammograms. Artificial Neural Networks.

Taylor, O. and MacIntyre, J. (1998). Adaptive local fusion systems for novelty detection and diagnostics in condition monitoring. In SPIE International Symposium on Aerospace/Defense Sensing.

Thompson, R. (1986). The neurobiology of learning and memory. Science, 233:941-947.

Thompson, R. and Spencer, W. (1966). Habituation: A model phenomenon for the study of neuronal substrates of behaviour. Psychological Review, 73(1):16-43.

Wang, D. and Arbib, M. A. (1992). Modelling the dishabituation hierarchy: The role of the primordial hippocampus. Biological Cybernetics, 76:535-544.

Wang, D. and Hsu, C. (1990). SLONN: A simulation language for modelling of neural networks. Simulation, 55:69-83.

Worden, K., Pierce, S., Manson, G., Philp, W., Staszewski, W., and Culshaw, B. (1999). Detection of defects in composite plates using lamp waves and novelty detection. International Journal of Systems Science.

Ypma, A. and Duin, R. P. (1997). Novelty detection using self-organizing maps. In Proceedings of International Conference on Neural Information Processing and Intelligent Information Systems. 